\title{
BEAM OPERATION OF THE SARAF LIGHT ION INJECTOR
}

\author{
Christian Piel, Kai Dunkel, Michael Pekeler, Hanspeter Vogel, Peter vom Stein, \\ ACCEL Instruments GmbH, Bergisch Gladbach, Germany
}

\section{Abstract}

In beginning of 2007 the installation of the first stage of SARAF has been finalized. The system consists out of an ECR ion source, a low energy beam transport system, a four rod RFQ, a medium energy transport system and a superconducting module housing 6 half resonators and three superconducting solenoids. This injector will be characterized with a diagnostic plate. The installation allows continuous measurement of beam charge, position and phase. The diagnostic plate in addition provides a beam halo monitor, vertical and horizontal slit and wire systems, a slow and a fast faraday cup, which can only be used in pulsed operation. The paper will describe the status of commissioning and will show first measurement results.

\section{ACCELERATOR DESIGN}

The $40 \mathrm{MeV}$ s. c. linac for protons and deuterons designed and currently built by ACCEL Instruments $\mathrm{GmbH}[1]$ is described in [2, 3]. It consists of an ECR ion source, a n. c. RFQ and six modules housing 46 s. c. half wave resonators. In the first phase of the project the source, the RFQ and a superconducting prototype module (PSM) with six superconducting resonators have been built and delivered. Currently the accelerator up to the RFQ including MEBT is under commissioning.

\section{ECR ION SOURCE AND LEBT}

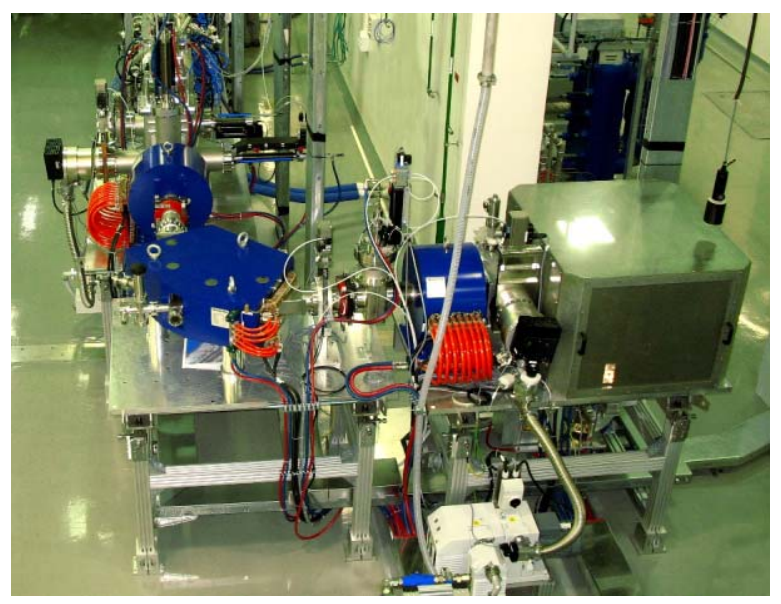

Figure 1: ECR Ion Source with LEBT installed at SARAF

The design of the ECR ion source based on a AECL design is described in [4] and consists of following subcomponents (figure 1):

- plasma chamber with accel/deceleration-electrodes

- gas flow system including dosing valve

- $2.45 \mathrm{GHz}$ magnetron providing required RF power

- two HV power supplies $(+20 /+40 \mathrm{kV}$ and $-5 \mathrm{kV})$
- two solenoid magnets defining the ECR zone

- LEBT including vacuum pumping, focussing, bending magnet and beam diagnostics

\section{Commissioning Results of Ion Source}

The ion source was assembled and commissioned at SARAF in 2006. It is operating with $\mathrm{H}+, \mathrm{H} 2+$ and $\mathrm{D}+$ at nominal currents and is under routine operation with proton beam for injector commissioning since March 2007. Details of commissioning results are described in [5].

For operation of the accelerator in a diagnostic mode (short beam pulses with low average beam power) the ion source can be pulsed with a pulse length in the millisecond range (figure 2).

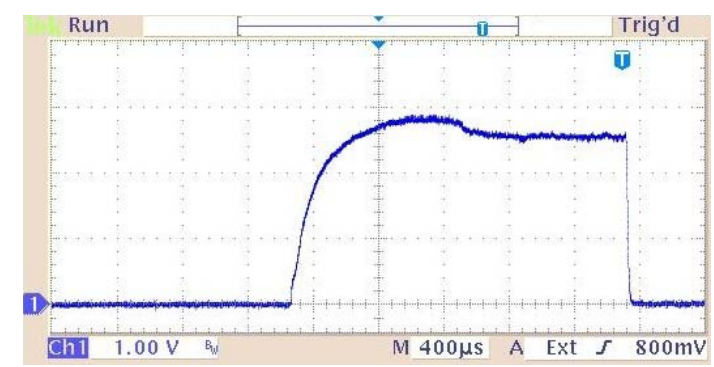

Figure 2: Ion Source in pulsed operation. Picture shows beam current measured at the LEBT Faraday Cup.

\section{RFQ ACCELERATOR}

The normal-conducting RFQ is designed to accelerate the ion beam in cw-mode from the ion source energy of $20 \mathrm{keV} / \mathrm{u}$ to $1.5 \mathrm{MeV} / \mathrm{u}$ being the input energy of the superconducting linac. The RFQ is conditioned up to a RF power of $200 \mathrm{~kW}$ in pulsed mode and $140 \mathrm{~kW}$ in cw mode, which is more than sufficient for proton operation at $\sim 60 \mathrm{~kW}$. After optimizing the proton beam operation the RFQ will be conditioned to the required power level for deuteron beam.

The LLRF of the RFQ keeps the amplitude constant within less than $1 \%$ and the phase stable better than $0.5^{\circ}$. This is still valid in the diagnostic mode when the RFQ power is pulsed. The timing of pulsing the ion source and the RFQ can be set in wide range of several seconds with a resolution of $50 \mathrm{~ns}$. The overlap of the two pulses defines the particle beam pulse length behind the RFQ in the diagnostic mode (figure 3 and 4), which is required to prevent beam intercepting diagnostics from damage due to high beam power. 


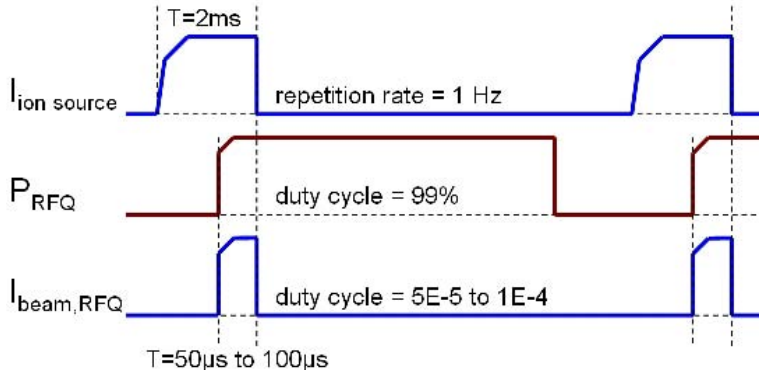

Figure 3: Timing diagram of the diagnostic mode for very low average beam power.

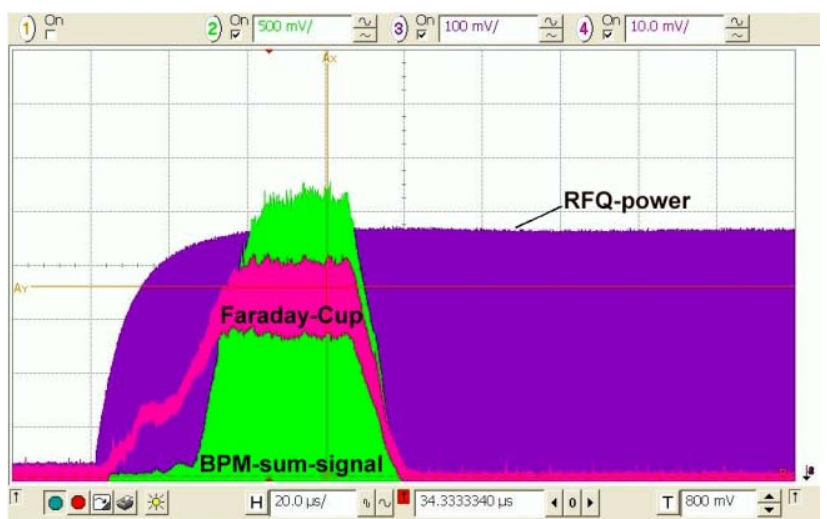

Figure 4: The usable beam pulse length in diagnostic mode is $\sim 30 \mu \mathrm{s}$

\section{MEDIUM ENERGY BEAM TRANSPORT}

The medium energy beam transport system is designed to match the beam transversal to the s. c. linac. It consists of following components, which are placed very compact within $650 \mathrm{~mm}$ (figure 5):

- $\quad$ three $31 \mathrm{~T} / \mathrm{m}$ quadrupole magnets each including two sets of steering magnets

- two 4-button beam position monitors (BPM) also providing beam phase signals

- two sets of X/Y-wire scanners for transversal beam profile measurements.

- $\quad$ vacuum equipment (three pumps and one gauge)

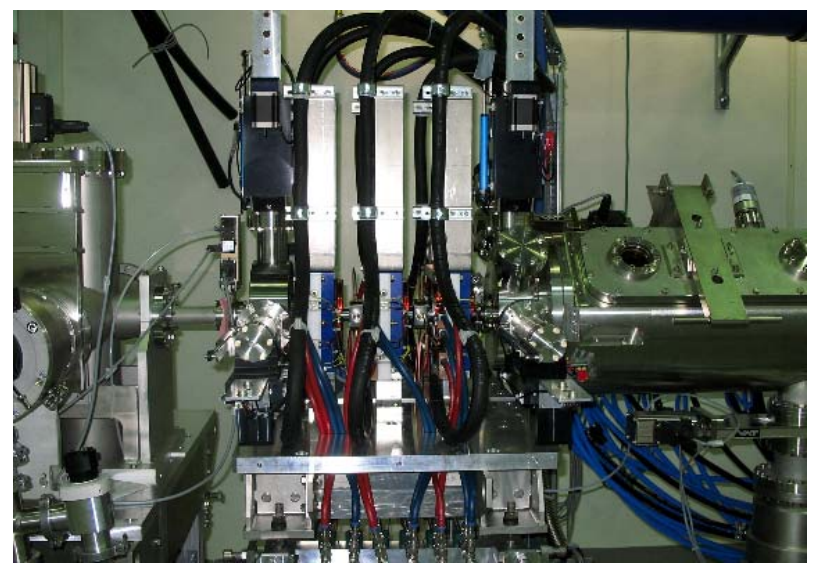

Figure 5: MEBT between RFQ (right) and D-plate (left)

\section{Commissioning Results of MEBT Diagnostics}

The sum signal of both BPM can be used as phase probes with a bandwidth of $500 \mathrm{MHz}$. With the known distance of $145 \mathrm{~mm}$ between the two BPM this setup can be used for time-of-flight (ToF) measurements to determine the beam energy (figure 6).

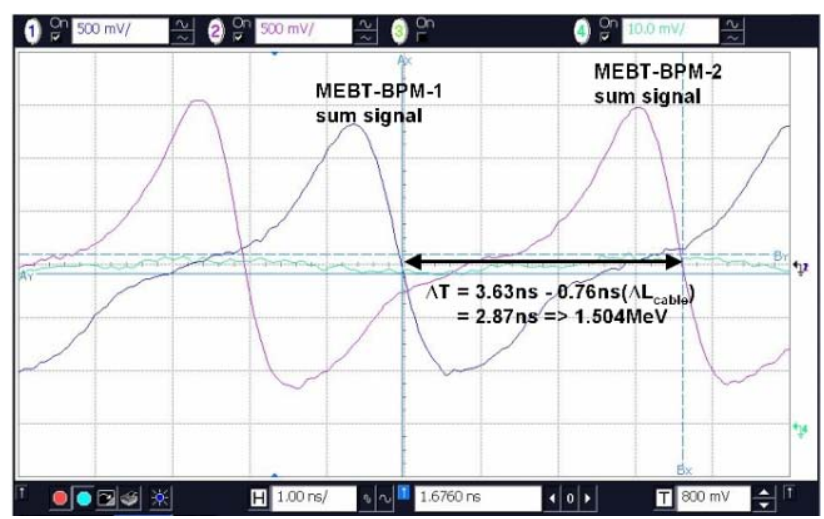

Figure 6: Time of flight measurement in MEBT with both BPM signals results in a beam energy of $1.504 \mathrm{MeV}+/-$ $12 \mathrm{keV}$.

The wire scanners are used to measure transversal beam profiles right after the RFQ and at the end of the MEBT to analyze the effects of quadrupole and steering magnets settings.

\section{DIAGNOSTIC PLATE}

The diagnostic plate (D-plate) was designed and built to analyze the beam performance of the RFQ and PSM as well as of the entire s. c. linac. For this purpose it is built on a moveable table as shown in figure 7 .

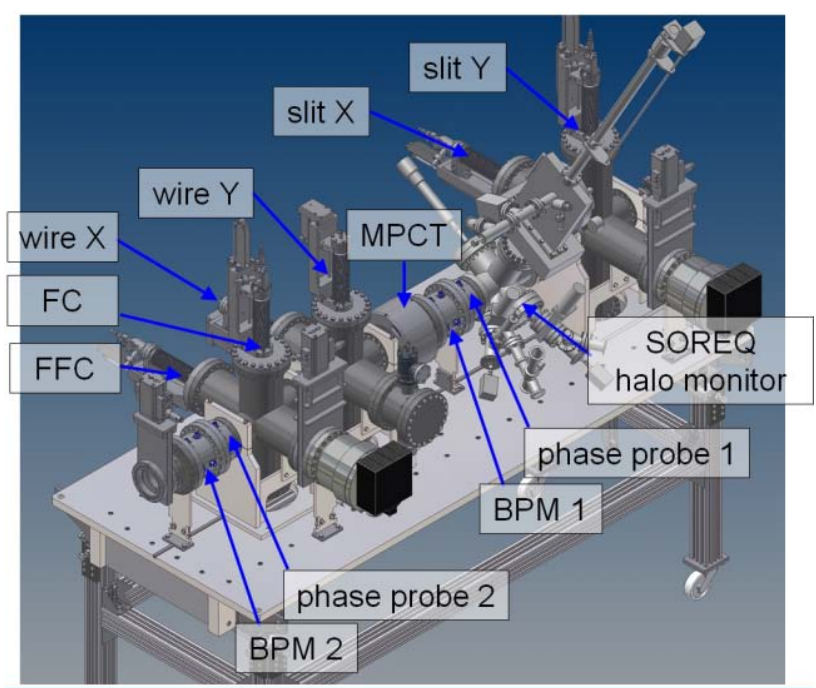

Figure 7: Layout of Diagnostic Plate

The D-plate consists of following components:

- a slit/wire system for transversal profile and emittance measurements ( $\mathrm{X}$ and $\mathrm{Y}$ axis),

- two phase probes for ToF measurements,

- two beam position monitors, 
- $\quad$ one current transformer (Bergoz MPCT) for nonintercepting current measurements,

- $\quad$ one biased Faraday-Cup

- a fast Faraday Cup (FFC) with a bandwidth of $6 \mathrm{GHz}$ for longitudinal beam profile measurements,

- halo monitor designed and built by SOREQ.

\section{Commissioning Results of Diagnostic Plate}

All diagnostic devices have been taken into operation. The wire scanners are used to measure transversal beam profiles in the D-Plate to analyze the effects of RFQ power (figure 7), quadrupole and steering magnets settings of the MEBT.
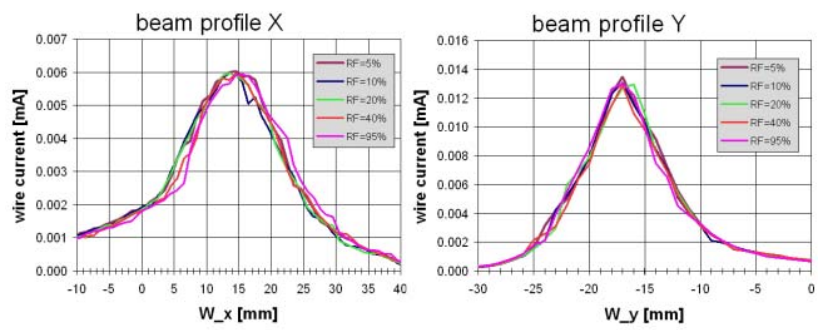

Figure 8: $\mathrm{X} / \mathrm{Y}$-profiles measured at different RFQ duty cycles show no influence on beam position or shape.

The phase probes in the D-Plate are used for ToF measurements. The resulting energy value showed excellent agreement with the MEBT measurement.

\section{Fast-Faraday-Cup}

The longitudinal profile measurement system consists of a $10 \mathrm{GHz}$ FFC, two $12 \mathrm{GHz}$ amplifiers, bias-T, low attenuation cable and a $6 \mathrm{GHz} / 40 \mathrm{GS} / \mathrm{s}$ real-time oscilloscope. The entire setup has a bandwidth of $6 \mathrm{GHz}$ and was analysed concerning the frequency response before delivery in order to make Fourier corrections to the measured bunch length.

The FFC allows to measure bunch lengths of $\sigma>26 \mathrm{ps}$ and together with a s. c. cavity of the PSM operated as a buncher longitudinal emittance measurements will be possible.

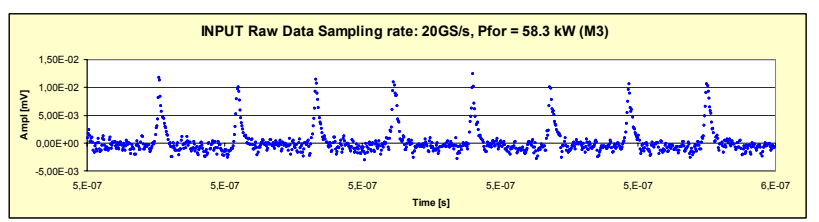

Figure 9: Raw data of measured longitudinal beam profiles of a bunch train.

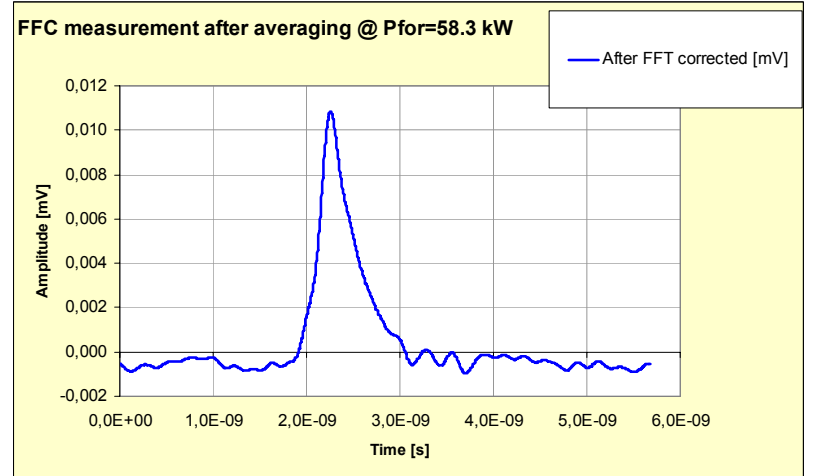

Figure 10: Measured longitudinal beam profile after averaging and Fourier correction.

\section{PROTOTYPE SUPERCONDUCTING MODULE}

The PSM is installed in the accelerator tunnel of SARAF beside the beamline of the accelarator and is connected to liquid helium supply and RF power. This setup allows testing of the cryogenic system and PSM RF-acceptance tests in parallel to the commissioning of the n. c. accelerator.

\section{OUTLOOK}

After the beam characterization of the normal conducting accelerator and finalization of cryogenic and RF tests for the superconducting cavities the PSM will be installed behind the n.c. accelerator. Beam commissioning of the entire injector including PSM is scheduled for late summer 2007.

\section{REFERENCES}

[1] http://www.accel.de

[2] M. Pekeler et al. "Design of a $40 \mathrm{MeV}$ linear accelerator for protons and deuterons using super conducting half wave resonators", EPAC'02, Paris, 2002

[3] K. Dunkel et al. "Custom design of medium energy linear accelerator systems”, EPAC’04, Luzern, 2005

[4] C.Piel et al. "Development and performance of a proton and deuteron ion source", PAC'05, Knoxville, 2005

[5] K. Dunkel et al. "Performance of the SARAF Ion Source", PAC'07, Albuquerque, 2007 